\title{
Hysteria, conversion and functional disorders: a neurological contribution to classification issues
}

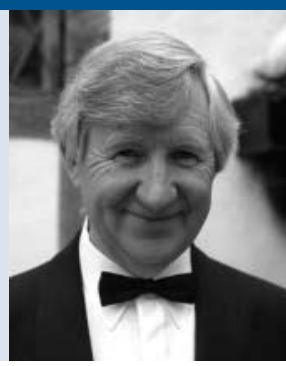

Edward H. Reynolds

\author{
Summary \\ Proposals by psychiatrists to reclassify conversion disorder \\ in DSM-5 and ICD-11 are proving difficult and controversial. \\ Patients with conversion disorder usually present initially \\ to neurologists, who often use different concepts and \\ terminology. History and clinical practice suggest that the \\ way forward is to seek agreed principles and a common
}

understanding between the two disciplines, preferably in a single universal classification.

\section{Declaration of interest}

None.
Edward H. Reynolds was formerly a consultant neurologist to the Maudsley and King's College Hospitals and Director of the Institute of Epileptology at King's College London.

The classifications of psychiatric disorders in DSM-IV ${ }^{1}$ and ICD $-10^{2}$ are both under review. In current classifications the traditional use of the term 'hysteria', based on the discredited theory of a wandering uterus, has been replaced by 'conversion disorder', influenced by the psychoanalytic theory of Freud, and by 'dissociative disorder', influenced by a related analytical theory of Janet. Within a review of somatoform disorders for DSM-5, proposals have been circulated to replace the term 'conversion disorder' with 'functional neurological disorder.' Similar suggestions are being discussed for ICD-11.

\section{Reported problems with 'conversion disorder'}

The arguments ${ }^{3,4}$ for abandoning the term 'conversion disorder' include: (a) it is based on a questionable psychoanalytical concept that is only one of several competing unproven aetiological hypotheses; (b) it is not widely used by non-psychiatrists and some assume it is not liked by patients; (c) the disorder should be viewed from a brain as well as a mind perspective, while avoiding an unhelpful brain-mind dichotomy; (d) the current 'conversion' diagnostic criteria require (i) a psychosocial association with symptom onset, which is difficult to find in a minority of patients, and when present is sometimes of questionable relevance; and (ii) the exclusion of feigning (malingering) which is difficult, if not impossible.

Kanaan et $a l^{5}$ broadly agree with the problems associated with the use of the term 'conversion disorder' and emphasise the difficulties in inserting the current concept, with its unique criteria of a psychosocial aetiology and absence of conscious deception, into any classification of somatoform disorders. Interestingly, however, although supporting a reclassification of 'conversion' patients as a whole as 'functional neurological symptoms' and dropping the requirement for a psychosocial association and exclusion of feigning, they do also propose that a subgroup with a determinate psychological explanation should retain the name 'conversion disorder', which they view as a healthy compromise.

\section{Problems with 'functional neurological disorder'}

There may be good reasons to dispose of or modify 'conversion disorder' but the alternative should also be well founded. Much less attention has been paid to the potential problems with the use of 'functional neurological disorder'. It is widely agreed that the clinical diagnosis of hysterical/conversion neurological symptoms, whether motor, sensory or convulsive, rests on the evidence of internal inconsistency or incongruity with any recognised neurological disorder. To a neurologist therefore it does not make sense to describe such inconsistent and incongruous symptoms as a neurological disorder.

There are even greater problems with the use of the term 'functional'. This is an ambiguous and confusing term with a long history and a diversity of meanings even among neurologists, 6,7 which led Kinnier Wilson to recommend its abolition as long ago as $1930 .^{8}$

A key feature of neurological symptoms in conversion disorder is that they defy our present and quite long understanding of neuroanatomy and neurophysiology. Patients have their own mental conceptions of right and left, of how motor or sensory function is distributed in a limb, of the function of muscle agonists and antagonists, and of the motor components of a seizure, which are quite different to those of a neurologist or a neurologically trained psychiatrist or physician. Furthermore, these neurological symptoms may remit dramatically with a variety of treatments such as physiotherapy, psychotherapy, hypnotism, electrical stimulation, persuasion, explanation, reassurance or no treatment at all, in a manner that seems inconsistent with any known recovery and repair mechanism in the nervous system. Of course, the onset and relapse of hysteria/ conversion symptoms may be sudden and dramatic but this is not unique and occurs in other neurological disorders.

None of this excludes the possibility of changes in nervous system function detectable by imaging or neurophysiological techniques in patients with conversion disorder. ${ }^{4}$ Many such patients have personality traits that include suggestibility, emotional instability or attention seeking. If, as has been long suspected, hysteria/conversion is psychological in origin, it would not be surprising if emotional nervous system pathways are involved. Indeed, vasomotor changes of presumed autonomic origin may be detectable in some paralysed limbs of hysterical origin. $^{8}$ 
Epilepsy and hysteria/conversion are universal disorders, perhaps as old as mankind itself. But whereas the variety and phenomena of seizures have not changed since Babylonian times, ${ }^{9}$ historians of hysteria have indicated that the clinical phenomena continue to vary with historical periods, ethnicity and culture. ${ }^{8,10}$ Epileptic seizures appear to be physiological reactions, truly a functional disorder of the nervous system, whether or not any pathology is present. Whereas, hysterical/conversion phenomena remain highly suspicious of psychological reactions that can also occur whether or not any underlying neuropathology is present, as for example, in multiple sclerosis or epilepsy. ${ }^{11}$ We know of many triggers for seizures, including emotional ones, but neurologists fail to find precipitants in a minority of patients, ${ }^{12}$ just as psychiatrists fail to find psychological stress or conflicts in hysteria/conversion in some of their patients. ${ }^{4,5}$

\section{An interdisciplinary approach to classification problems}

Two separate and uncoordinated groups of psychiatrists grapple with the classification issues in DSM and ICD with relatively little input from neurologists. There are no agreed principles of psychiatric classification, for example phenomenological, aetiological, prognostic or patient preference, the latter a relatively recent criterion, ${ }^{13}$ but historically the terms hysteria, conversion and dissociation have been based on aetiological concepts.

In clinical practice in most advanced economies, patients with hysteria/conversion disorder eventually present to neurologists who usually make the diagnosis. Neurologists tend to use their own terminology and concepts, commonly 'functional', but with a variety of meanings, most often 'non-organic.' Patients are then commonly referred on by neurologists to psychiatrists, with their own terminology, for aetiological clarification and treatment, quite often unsuccessfully on one or both counts. Thus, many patients fall between the two disciplines ${ }^{5,7}$ while acquiring additional conceptual and terminological confusion.

In future there should be an interdisciplinary approach to classification with comparable neurological involvement, preferably in one, not two, universal classifications, so that psychiatrists and neurologists can attempt to agree common principles and terminology, including bearing patient acceptance in mind. Despite some apparent loss of confidence in psychological theories, it would be unwise to abandon current classifications of either conversion or dissociative disorders, or both, until some common understanding and agreement for alternatives are reached between the two disciplines. The previous switch from hysteria to conversion and dissociation illustrates the danger. The starting point for discussion should be that the symptoms are neurological but of psychogenic origin; this would leave open the largely uncharted field of the psychophysiological mechanisms of the symptoms and the continuing uncertainty about the probably multiple possible psychological aetiologies.

Edward H. Reynolds, MD, FRCP, FRCPsych, Department of Clinical Neurosciences, King's College School of Medicine, Weston Education Centre, Denmark Hill Campus, Cutcombe Road, London SE5 6PJ, UK. Email: reynolds@buckles.u-net.com

First received 6 Mar 2012, final revision 11 Jun 2012, accepted 21 June 2012

\section{Acknowledgements}

I am grateful to $\mathrm{Dr}$ T. Nicholson for drawing my attention to this debate and for constructive discussion; and to Dr J. Stone for constructive discussion.

\section{References}

1 American Psychiatric Association. Diagnostic and Statistical Manual of Mental Disorders (4th edn) (DSM-IV). APA, 1994.

2 World Health Organization. The ICD-10 Classification of Mental and Behavioural Disorders: Clinical Descriptions and Diagnostic Guidelines. WHO, 1992.

3 Dimsdale J, Creed F. The proposed diagnosis of somatic symptom disorders in DSM-V to replace somatoform disorders in DSM-IV - a preliminary report. J Psychosom Res 2009; 66: 473-6.

4 Stone J, Lafrance WC, Levenson JL, Sharpe M. Issues for DSM-5: conversion disorder. Am J Psychiatry 2010; 167: 626-7.

5 Kanaan RA, Carson A, Wessely SC, Nicholson TR, Aybek S, David AS. What's so special about conversion disorder? A problem and a proposal for diagnostic classification. Br J Psychiatry 2010; 196: 427-8.

6 Reynolds EH. Structure and function in neurology and psychiatry. Br J Psychiatry 1990; 157: 481-90.

7 Kanaan RA, Armstrong D, Wessely SC. The function of 'functional': a mixed methods investigation. J Neurol Neurosurg Psychiatry 2012; 83: 248-50.

8 Kinnier Wilson SA. The approach to the study of hysteria. Proc $R$ Soc Med 1930; 24: 173-84.

9 Kinnier Wilson JV, Reynolds EH. Translation and analysis of a cuneiform text forming part of a Babylonian treatise on epilepsy. Med Hist 1990; 34: 185-98.

10 Veith I. Hysteria: The History of a Disease. University of Chicago Press, 1965.

11 Slater ET. Diagnosis of 'hysteria'. BMJ 1965; 1: 1395-9.

12 Frucht MM, Quigg M, Schwaner C, Fountain NB. Distribution of seizure precipitants among epilepsy syndromes. Epilepsia 2000; 41: 1534-9.

13 Stone J, Wojcik W, Durrance D, Carson A, Lewis S, MacKenzie L, et al. What should we say to patients with symptoms unexplained by disease? The 'number needed to offend'. BMJ 2002; 325: 1449-50. 